\title{
Effect of cable load impedance on coupling schemes for MV power line communication
}

Citation for published version (APA):

Wouters, P. A. A. F., Wielen, van der, P. C. J. M., Veen, J., Wagenaars, P., \& Steennis, E. F. (2005). Effect of cable load impedance on coupling schemes for MV power line communication. IEEE Transactions on Power Delivery, 20(2), 638-645. https://doi.org/10.1109/TPWRD.2005.844334

DOI:

10.1109/TPWRD.2005.844334

Document status and date:

Published: 01/01/2005

\section{Document Version:}

Publisher's PDF, also known as Version of Record (includes final page, issue and volume numbers)

\section{Please check the document version of this publication:}

- A submitted manuscript is the version of the article upon submission and before peer-review. There can be important differences between the submitted version and the official published version of record. People interested in the research are advised to contact the author for the final version of the publication, or visit the $\mathrm{DOI}$ to the publisher's website.

- The final author version and the galley proof are versions of the publication after peer review.

- The final published version features the final layout of the paper including the volume, issue and page numbers.

Link to publication

\section{General rights}

Copyright and moral rights for the publications made accessible in the public portal are retained by the authors and/or other copyright owners and it is a condition of accessing publications that users recognise and abide by the legal requirements associated with these rights.

- Users may download and print one copy of any publication from the public portal for the purpose of private study or research.

- You may not further distribute the material or use it for any profit-making activity or commercial gain

- You may freely distribute the URL identifying the publication in the public portal.

If the publication is distributed under the terms of Article 25fa of the Dutch Copyright Act, indicated by the "Taverne" license above, please follow below link for the End User Agreement:

www.tue.nl/taverne

Take down policy

If you believe that this document breaches copyright please contact us at:

openaccess@tue.nl

providing details and we will investigate your claim. 


\title{
Effect of Cable Load Impedance on Coupling Schemes for MV Power Line Communication
}

\author{
Peter A. A. F. Wouters, Peter C. J. M. van der Wielen, Student Member, IEEE, Jeroen Veen, Student Member, IEEE, \\ Paul Wagenaars, and E. Fred Steennis
}

\begin{abstract}
Coupling of carrier wave frequencies up to $95 \mathrm{kHz}$ (within the European CENELEC A-band) for online diagnostic data transfer in medium voltage cables is studied. Inductive and capacitive signal coupling is considered not only on basis of technical performance, but also on basis of practical aspects. The effectiveness of coupling schemes depends on the impedances of substation equipment at the cable terminals. The frequency response of a 10-kV, 400-kVA three-phase cast resin distribution transformer is investigated. In the frequency range of interest, the behavior is well described by a capacitance of typically $1 \mathrm{nF}$. The signal transfer over a 4-km paper cable, terminated by various load impedances to mimic real equipment is studied. From the results it is concluded that for inductive coupling performance within the CENELEC $A$-band may be sufficient, except for substations at the end of a grid. Transferring signals containing frequencies up to several megahertz, which is already required for synchronization of partial discharge detection and location equipment, is feasible under all conditions. Measurements on life substations indicate that up to these frequencies substation components can still be accurately modeled as lumped circuit impedances.
\end{abstract}

Index Terms-Communication channels, fault location, multi-conductor transmission lines, noise measurement, partial discharges, power cable insulation, power transformers, propagation, substation measurements.

\section{INTRODUCTION}

A POSSIBLE application of power line communication for utilities is the transfer of data concerning the condition of the medium voltage (MV) grid. For future intelligent MV grids data concerning the state of equipment, momentary load-flow, etc. must be transferred between substations within the grid. More specifically, the authors' interest involves transmission of diagnostic information from online partial discharge (PD) measurements [1]. For this purpose the amount of data is not expected to be a decisive issue, and in principle the CENELEC $A$-band available for utilities $(9-95 \mathrm{kHz}$; European standard EN50065) allows a sufficient data transfer rate. In contrast, widespread Internet application via the MV grid seems unlikely due to the carrier frequency restrictions.

Available power cable diagnostics include PD measurements on temporally disconnected connections, which are externally

Manuscript received February 23, 2004. This work was supported by KEMA Nederland B.V., Arnhem, The Netherlands, as part of the TDP24 project. This paper is based on a contribution presented by the authors at the 2003 IEEE Bologna PowerTech, Bologna, Italy, June 23-26, 2003. Paper no. TPWRD00066-2004.

The authors are with Eindhoven University of Technology, 5600 MB Eindhoven, The Netherlands (e-mail: p.a.a.f.wouters@tue.nl; p.c.j.m.v.d.wielen@tue.nl; j.veen@tue.nl; $\quad$ p.wagenaars@student.tue.nl; e.f.steennis@tue.nl).

Digital Object Identifier 10.1109/TPWRD.2005.844334

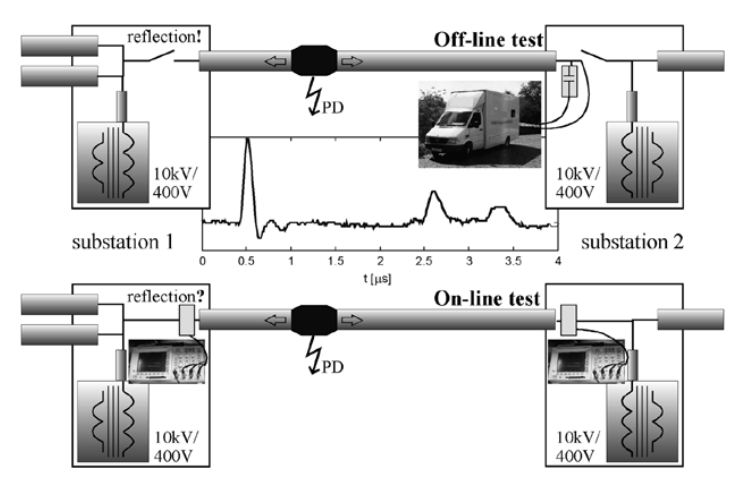

Fig. 1. Situation during off-line (top) and online (bottom) tests. For off-line PD testing the cable is disconnected from the grid and the cable is energized externally. The PD pulses (e.g., originating from a cable joint) traveling in both directions can be detected at one side, because of full signal reflection at the far end (see inserted waveform). Signal reflection at the far end in an online test depends on the substation configuration, and TDR may be prohibited. Location can now be realized by double-sided detection.

energized [Fig. 1(top)]. The PD events result in pulses, which travel in both directions along the cable. Since the far cable end is open, full signal reflection takes place and the origin of the PD can be established by means of time domain reflectometry (TDR). From an operational point of view and also considering the costs involved, online techniques are preferred [Fig. 1(bottom)] [1]-[3]. More importantly, online diagnostics allow detection of trends in PD behavior over long periods of time and may therefore be more conclusive than offline tests. However, no clear signal reflection is guaranteed, since substation impedances now load the far end of the cable. Two-sided detection can be used instead of TDR. Linking the data from both sides allows accurate PD location, but requires information transfer. An online PD detection and location system therefore should preferably include a communication channel.

At present, substations at the MV level are generally not equipped with provisions for communication. Therefore, the grid itself is considered as an alternative channel. The main obstacle for using MV cables is related to the coupling into the grid. In this respect, the situation is completely opposite to power line communication over the low voltage grid, where coupling is relatively easy but cable branching and connected loads are responsible for severe signal attenuation.

MV cables are shielded structures except for their connections in substations. There, signals can be coupled to the grid basically in two ways.

- Capacitive coupling requires a high-voltage capacitor connected to one of the phase conductors. This complicates installation, especially in a life circuit. 


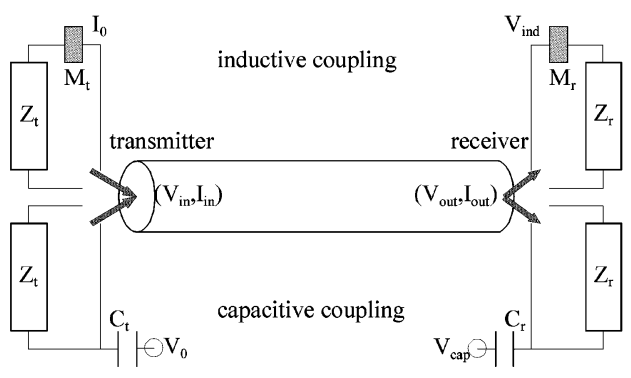

Fig. 2. Inductive coupling ( $I_{0}$ : injected current, $V_{\text {ind }}$ : detected induction voltage) and capacitive coupling ( $V_{0}$ : injected voltage, $V_{\text {cap }}$ : detected voltage). The mutual inductances at transmitter and receiver side are denoted with $M_{t}$ and $M_{r}$, respectively; the capacitive coupling is realized through $C_{t}$ and $C_{r}$. The voltage and current at the cable input and output are denoted with $V_{\text {in }}, I_{\text {in }}$ respectively $V_{\text {out }}, I_{\text {out }}$.

- Inductive coupling does not require galvanic contact with the phase conductors. However, a suitable injection site must be accessible.

Especially for inductive coupling, the impedances present in a substation are essential, since they close the current injection and detection circuits. Besides for communication, these impedances are relevant for PD magnitude detection, and also for synchronization. The time-base of equipment at both cable ends must be aligned at least within 100 ns to allow sufficient location accuracy (about 10-20 m). For this purpose, the option of pulse injection over the grid is investigated.

In this paper, the load of a typical distribution transformer, and the transmission of signals along a 4-km three-phase belted cable are studied in the frequency range of the CENELEC A-band. Although inductive coupling is preferred from a practical point of view, the effect of the termination impedance on capacitive coupling is studied as well in order to compare various (combinations of) coupling schemes. The results are used to evaluate the channels' performance for both carrier wave (up to $100 \mathrm{kHz}$ ) coupling and coupling of PD synchronization signals (up to a few megahertz) in energized cable connections.

\section{TRANSMISSION LINE DESCRIPTION}

A survey of MV systems used in The Netherlands showed that for the majority of the substations, coupling sites are accessible for injecting or detecting signals by placing a coil either around a phase conductor at a cable end (where the earth screen is still present), or around the earth connection nearby the cable [4]. This inductive coupling scheme is basically a weakly coupled transformer. The coil is the primary side and the secondary side consists of the cable in series with the terminating impedance $Z_{t, r}$, which represents all substation components, e.g., distribution transformer, leaving cables (Fig. 2, transmission side: index $t$; receiver side: index $r$ ). For inductive coupling this impedance is preferably low. In contrast, this impedance should be high for capacitive coupling because it acts as a load for the transmitter.

The relevant impedances for a three-phase belted paper cable with a symmetrical load (e.g., a transformer) are schematically shown in Fig. 3. With the assumed three-fold symmetry the impedances of both cable and load can be described by only two (complex) parameters, namely the impedance between the
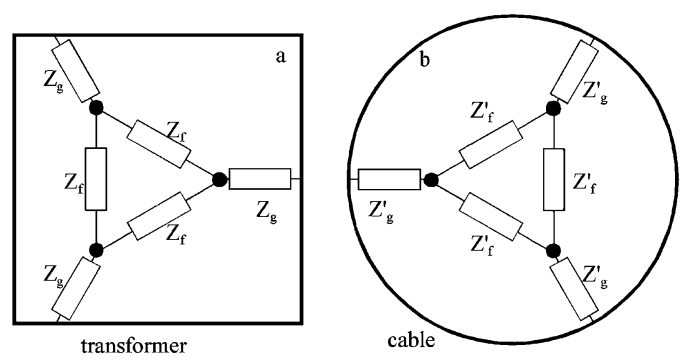

Fig. 3. Representation of (a) a three-phase transformer and (b) a three-phase cable as lumped components for a three-fold rotation symmetrical system. $Z_{f}$ and $Z_{f}^{\prime}$ represent the impedances between the phases; $Z_{g}$ and $Z_{g}^{\prime}$ the shield to phase impedances.
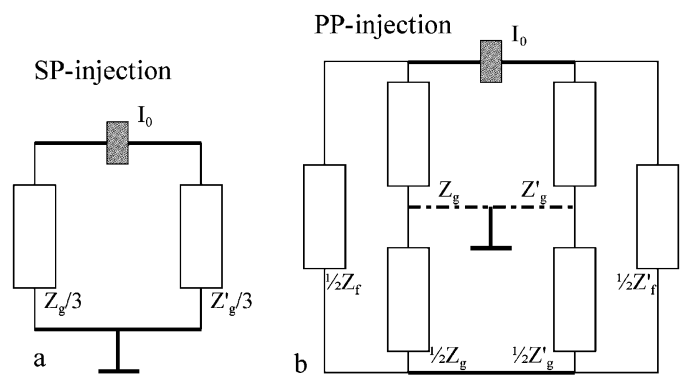

Fig. 4. Equivalent input circuit for inductive coupling to three phases with respect to ground (a) SP-mode, and to one phase with respect to the other phases (b) PP-mode with SP fraction in case the return current flows partly through ground).

phases (index $f$ ) and the impedance with respect to ground (index $g$ ). Two modes of signal coupling can be distinguished [5], [6] as follows.

- Shield to phase mode (SP): the signal is injected in all three phases, and the return path is the ground connection. The current loop is closed by impedances between the phases and ground, as indicated in Fig. 4(a).

- Phase to phase mode (PP): The signal is injected between two phases. If the signal is injected over only one phase conductor, also the ground connection is a return path resulting in an additional SP component, see Fig. 4(b).

For simplicity, we will consider the circuit effectively as a two-conductor transmission line, avoiding introduction of matrices to describe the effects at the cable ends [7]. For the cable connection (characterized by impedance, $Z_{0}$, propagation coefficient, $\gamma(\omega)$, and length, $\ell$ ) shown in Fig. 2 the transfer functions of the four possible combinations of inductive (index $i$ ) and capacitive (index $c$ ) coupling are given by

$$
\begin{aligned}
H_{i-i} \equiv & \frac{I_{\text {out }}}{I_{0}}=\frac{1}{2} \frac{j \omega M_{t}}{Z_{0}} \cdot \frac{Z_{0} \tau_{t}(\omega)}{Z_{t}(\omega)} \cdot \frac{Z_{0} \tau_{r}(\omega)}{Z_{r}(\omega)} \\
& \cdot \frac{\mathrm{e}^{-\gamma(\omega) \ell}}{1-\rho_{t}(\omega) \rho_{r}(\omega) \mathrm{e}^{-2 \gamma(\omega) \ell}} \\
H_{i-c} \equiv & \frac{V_{\text {out }}}{I_{0}}=\frac{1}{2} j \omega M_{t} \cdot \frac{Z_{0} \tau_{t}(\omega)}{Z_{t}(\omega)} \cdot \tau_{r}(\omega) \\
& \cdot \frac{\mathrm{e}^{-\gamma(\omega) \ell}}{1-\rho_{t}(\omega) \rho_{r}(\omega) \mathrm{e}^{-2 \gamma(\omega) \ell}} \\
H_{c-i} \equiv & \frac{I_{\text {out }}}{V_{0}}=\frac{1}{2} j \omega C_{t} \cdot \tau_{t}(\omega) \cdot \frac{Z_{0} \tau_{r}(\omega)}{Z_{r}(\omega)}
\end{aligned}
$$


TABLE I

Signal Coupling With THE MV CABLE FOR INDUCTIVE COUPLING UPON CURRENT $I_{0}$ AND FOR CAPACITIVE COUPLing Upon Voltage $V_{0}$. At THE TransmitTER Side, $I_{\text {in }}$ IS THE CURRENT THROUGH AND $V_{\text {in }}$ IS THE Voltage Over the CABle ImPEDANCE. At THE ReCEIVER Side the Voltage $V_{\text {out }}$ AND CURRENT $I_{\text {out }}$ AT THE CABLE END RESUlT IN VolTAGES $V_{\text {ind }}$ AND $V_{\text {cap }}$ FOR INDUCTIVE AND CAPACITIVE DETECTION, RESPECTIVELY

\begin{tabular}{l|l|l}
\hline & Inductive coupling & Capacitive coupling \\
\hline Injection & $\frac{I_{\text {in }}}{I_{0}}=\frac{j \omega_{0} M_{t}}{Z_{0}+Z_{t}}$ & $\frac{V_{\text {in }}}{V_{0}}=j \omega_{0} C_{t}\left(Z_{0} / / Z_{t}\right)$ \\
\hline Detection & $\frac{V_{\text {ind }}}{I_{\text {out }}}=j \omega_{0} M_{r} Q$ & $\frac{V_{\text {cap }}}{V_{\text {out }}}=1$ \\
\hline
\end{tabular}

$$
\begin{aligned}
& \cdot \frac{\mathrm{e}^{-\gamma(\omega) \ell}}{1-\rho_{t}(\omega) \rho_{r}(\omega) \mathrm{e}^{-2 \gamma(\omega) \ell}} \\
H_{c-c} \equiv & \frac{V_{\text {out }}}{V_{0}}=\frac{1}{2} j \omega C_{t} Z_{0} \cdot \tau_{t}(\omega) \cdot \tau_{r}(\omega) \\
& \cdot \frac{\mathrm{e}^{-\gamma(\omega) \ell}}{1-\rho_{t}(\omega) \rho_{r}(\omega) \mathrm{e}^{-2 \gamma(\omega) \ell}}
\end{aligned}
$$

The set of equations in (1) relate the applied excitation current $I_{0}$ (inductive coupling) or excitation voltage $V_{0}$ (capacitive coupling) to the output current $I_{\text {out }}$ through (inductive coupling), or output voltage $V_{\text {out }}$ over (capacitive coupling) impedance $Z_{r}$. In (1) it is assumed that the self-induction of the input and output circuits are negligible, and that the impedance of $C_{t}$ is high with respect to $Z_{0}$ for the frequencies of interest.

The voltage reflection coefficient $\rho$ and transmission coefficient $\tau$ are defined by

$$
\rho_{t, r}=\frac{Z_{t, r} / Z_{0}-1}{Z_{t, r} / Z_{0}+1}, \quad \tau_{t, r}=\rho_{t, r}+1
$$

The four factors (separated by dots) in (1) can be interpreted as (i) the coupling strength at the input, the transmission factors at (ii) the transmitter and (iii) the receiver side, and (iv) a factor describing the signal propagation delay including attenuation, dispersion and (multiple) reflections.

The actual detected voltage, either capacitively $\left(V_{\text {cap }}\right)$ or inductively $\left(V_{\text {ind }}\right)$, depends on details of the detection circuit, which are not included in (1). For capacitive coupling the receiver had a high input impedance and did not load the system. For inductive coupling, a resonant circuit around a chosen detection frequency $\omega_{0}$ with quality factor $Q$ was used. The transfer functions at the injection and detection side are summarized in Table I.

\section{RESULTS FOR A 400-kVA TRANSFORMER}

The impedances arising in Figs. 3 and 4 are determined for a 400-kVA, 10-kV/380 V cast resin transformer using a vector impedance bridge. The impedance $Z_{g}$ from phase to ground can be described as a (stray) capacitance $C_{g}$. The impedance between two phases is modeled as an inductance $L_{f}$ parallel to a stray capacitance $C_{f}$. The inductance $L_{f}$ corresponds to the equivalent inductance of the primary coils. Its value will depend on the load at the low-voltage side. Therefore, the impedances were measured both with the low-voltage side open and short-circuited. The results for three of the tested configurations are shown in Fig. 5: (a) impedance between three connected phases and ground (as in Fig. 4(a)), (b) impedance between one phase and ground with the other phases floating (as in Fig. 4(b)), and (c) impedance between two phases. The latter configuration corresponds to coupling by two identical coils around two phases exciting one single PP-mode. The $1 / f$ behavior of the absolute impedance and the argument equal to $-90^{\circ}$ in Fig. 5(a) indicate a dominant capacitive behavior over the full frequency range. In Figs. 5(b) and (c) LC-resonances from the capacitances with the coil inductance are present. Since the equivalent inductance depends on the situation at the low-voltage side (open or short-circuit), the resonance frequencies are different for both extreme situations. At the resonances, the argument switches suddenly between $-90^{\circ}$ and $90^{\circ}$ because the resistive losses were negligible.

The curves in Fig. 5 are fitted using the following parameters: $C_{g}=315 \mathrm{pF}, C_{f}=260 \mathrm{pF}$, and $L_{f}=17 \mathrm{H}$ or $0.086 \mathrm{H} \mathrm{de}-$ pending on the low-voltage side. Although the transformer had in fact a linear coil arrangement, the description according to the symmetry of Fig. 3 gives good results. The three coils are equal and apparently the parasitic capacitive coupling is similar, probably because it is mainly determined by the capacitance between coils and core. For frequencies above about $50 \mathrm{kHz}$ the transformer behaves like a capacitor under all conditions. The total impedance by which the transformer loads a cable according to either the SP or PP scheme, taking the various parallel operating channels into account, corresponds to a value close to $1 \mathrm{nF}$. For oil-insulated transformers in metal enclosures a higher capacitance can be expected. Moreover, the capacitance of the transformer connections must be included. The value of $1 \mathrm{nF}$ can probably be regarded as a lower limit.

\section{Test on A 4-km MV CABle Connection}

A 4-km three-phase belted Paper-Insulated Lead-Covered (PILC) cable connection was used to study the signal transmission properties. The cable was loaded at both sides with various impedances, resistive as well as capacitive, to mimic different substation configurations. Inductive coupling was performed using an air coil (Rogowski) with a mutual inductance of $1.9 \mu \mathrm{H}$; a $2 \mathrm{nF}$ capacitor was used for capacitive coupling. A carrier wave was injected between one phase and ground with the second phase grounded and the third phase floating (due to lack of space for yet another ground connection). In Fig. 6 details of the experimental arrangement are shown. In order to characterize a three-phase load, the termination impedances were chosen such that they simulate the situation for three-phase operation. The results obtained at a frequency of $80 \mathrm{kHz}$ are shown in Fig. 7 for the four possible combinations of inductive and capacitive coupling. The lines indicate the calculated responses according to (1) and the coupling strengths from Table I using the following assumptions:

- Skin effect dominates the signal attenuation, $\alpha(\omega)$, the real part of the propagation coefficient, $\gamma(\omega)$. The attenuation is therefore proportional to $\sqrt{ } \omega$. 

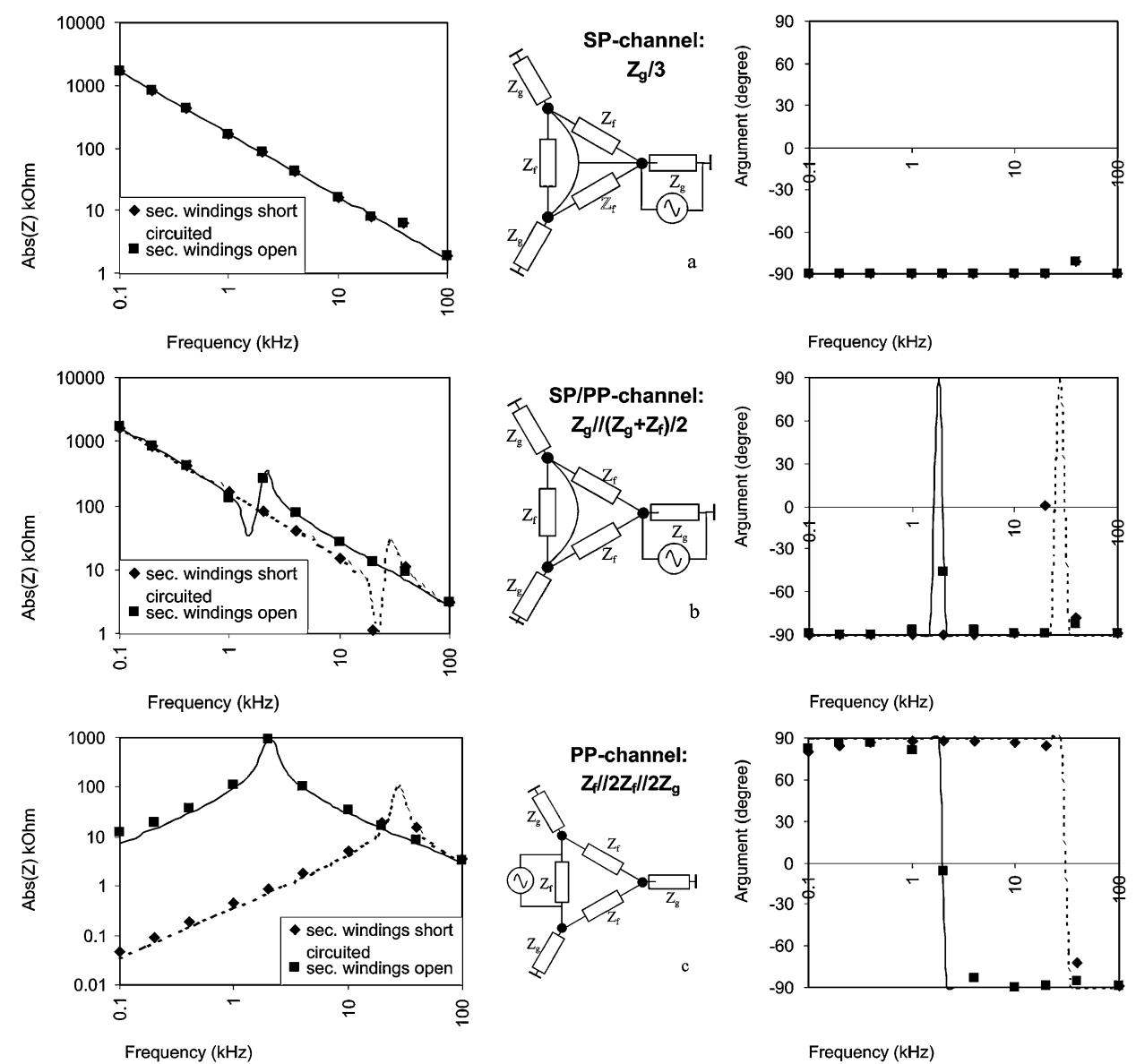

Fig. 5. Transformer impedance (absolute value and argument) between $100 \mathrm{~Hz}$ and $100 \mathrm{kHz}$ for three situations: (a) three phases connected with respect to ground (SP-mode), (b) one phase with respect to ground, and (c) between two phases (PP-mode). The solid line corresponds to an open transformer low-voltage side; the broken line corresponds to a short circuit at the low-voltage side.

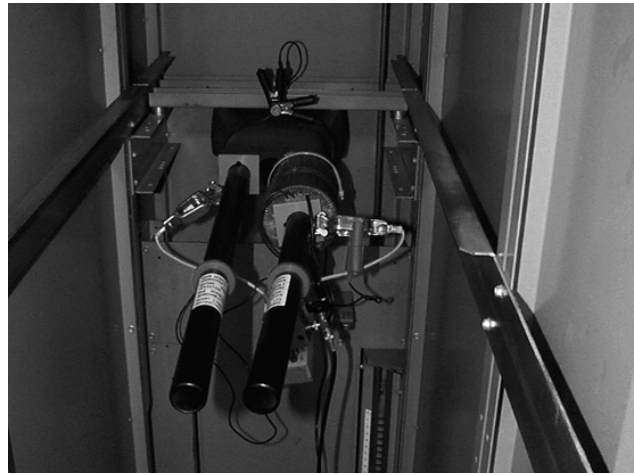

Fig. 6. Injection coil installed at one end of a $4 \mathrm{~km}$ PILC cable.

- Attenuation over 4-km cable is sufficient to neglect the effect of reflections back and forth the cable. The denominator in the last factor of (1) can therefore be set to one.

Two free parameters remain for curve fitting in all situations tested (also at other frequencies). The cable characteristic impedance was taken equal to $40 \Omega$, and the attenuation factor, $\alpha(\omega) \ell$, was set to $0.0010 \cdot \sqrt{ } \omega\left(\omega\right.$ in $\left.\mathrm{s}^{-1}\right)$. The model describes the experimental data well, except for some deviation in case of the $1 \mathrm{nF}$ termination when the signal is inductively coupled out. This may be ascribed to neglecting the measuring cable
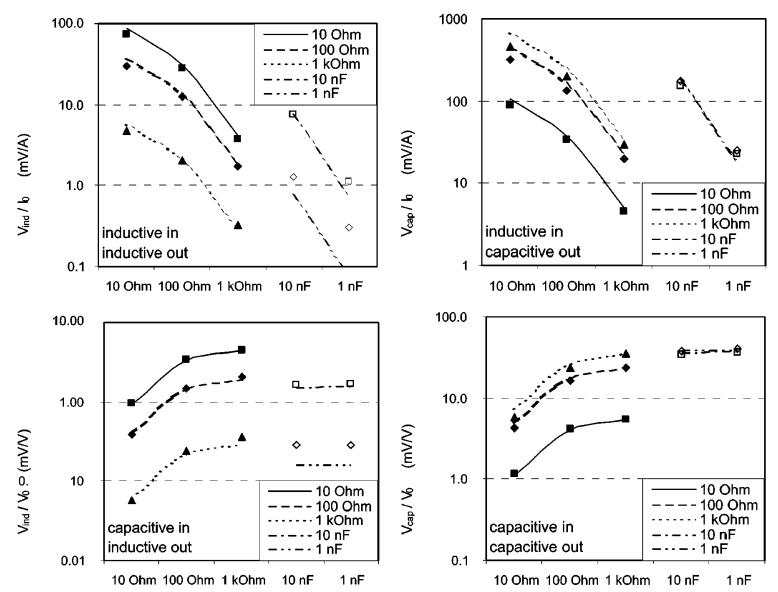

Fig. 7. Measured (symbols) and calculated (lines) signal transfer measured at $80 \mathrm{kHz}$ for the four combinations of inductive and capacitive coupling as a function of the impedances at the transmitter side $Z_{t}$ (along the horizontal axis) and receiver side $Z_{r}$ (indicated with labels).

capacitance connected to the termination impedance. The results basically confirm the importance of the cable termination: inductive coupling gives rise to relatively high signal levels only when the termination impedance is low, whereas for capacitive coupling, high impedances are preferred. 

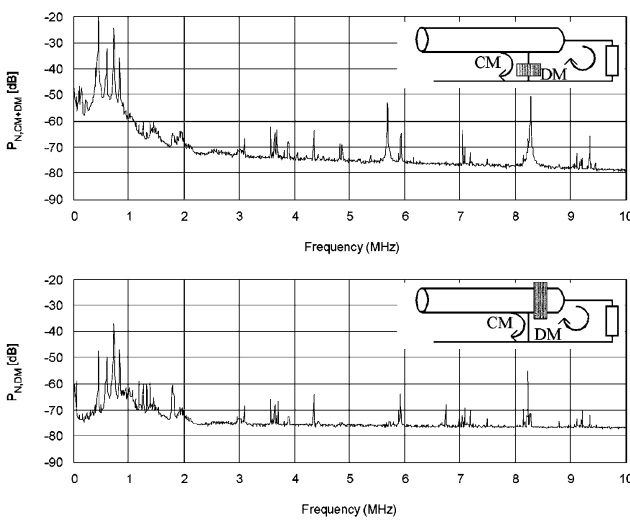

Fig. 8. Power spectrum density function from two different positions at a cable terminal (see Fig. 9). Top: signal detected at the earth connection to the cable earth screen (CM+DM component); bottom: signal detected at the cable shield, but beyond the earth connection (only DM component).

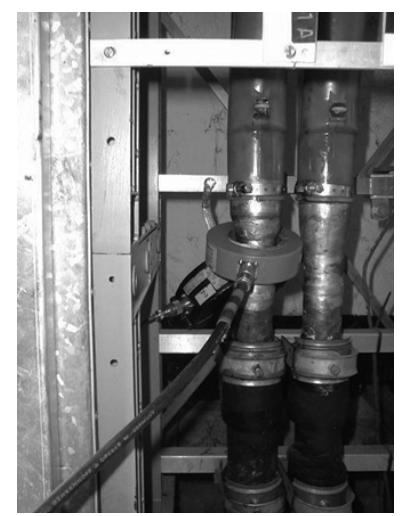

Fig. 9. Commercial inductive coil placed around the cable screen beyond the earth connection. A second sensor at the back (left) is placed around the earth connection to the cable screen.

\section{NOISE LEVEL}

Sixteen cables in life circuits from two Dutch utilities have been tested at both sides to determine the noise level [4]. Commercially available inductive sensors with a bandwidth of several tens of megahertz were positioned at various locations interesting for PD detection, and accessible also for power line communication. The results for two sensor positions, which are shown in the inserts of Fig. 8, are of particular interest:

- Inductive coupling to the connection between earth screen and ground (top). Safe installation without disrupting the power is possible here. However, besides the differential mode (DM) current involving the signals of interest, a common mode (CM) current is detected, which only contains interference from the outside world.

- Inductive coupling to the conductors (bottom). If the coupling takes place beyond the last earth connection, only the DM current is detected. For safe installation the cable screen must extend beyond the sensor position. For a large fraction of installations used in The Netherlands this can be realized as shown in Fig. 9.

The noise power spectrum density functions, see e.g., [8], in Fig. 8 clearly indicate that coupling involving only the DM channel is preferred if it is technically possible. The noise level
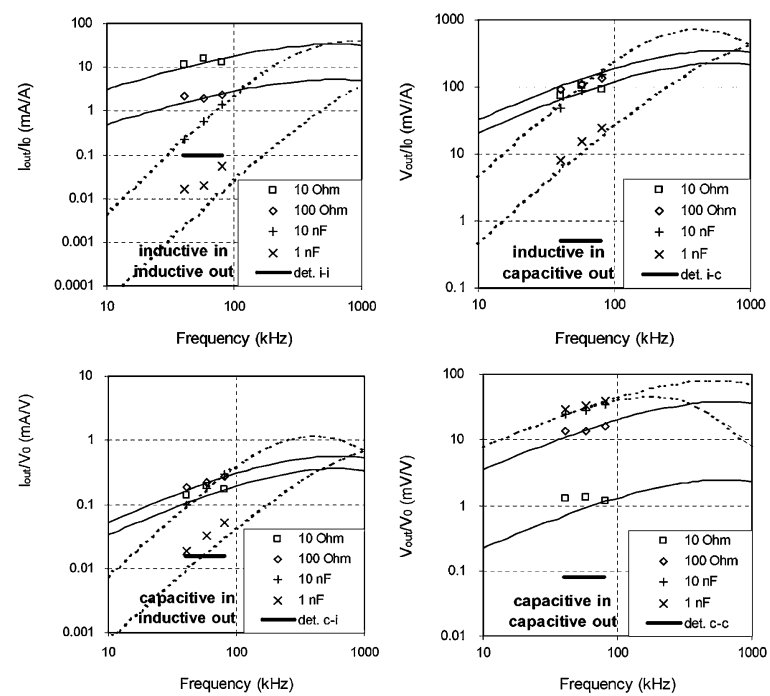

Fig. 10. Extrapolation of the response according to (1) up to $1 \mathrm{MHz}$ for the coupling schemes at different load conditions at both cable ends. The symbols are obtained from the measured data and serve as "calibration points" for the theoretical curves. The bold lines indicate the sensitivity of a FSK (Frequency Shift Keying) communication device.

is about $20 \mathrm{~dB}$ better compared to the channel also containing the $\mathrm{CM}$ channel for the carrier frequency range allowed by the standards.

\section{COUPLING EFFICIENCY}

Since transformers tend to behave as a capacitor in the frequency range of interest, it is expected that inductive coupling becomes more competitive at increasing frequency.

In Fig. 10 the theoretical behavior of (1) is plotted in the frequency range from $10 \mathrm{kHz}$ to $1 \mathrm{MHz}$ using the parameters determined in Section IV. Hereby it is assumed, that the main cable losses are caused by the skin effect and the transformer can still be described as a capacitor. However, at higher frequencies dielectric losses in the cable can start to become important, and also the transformer may show some resonances [9]. The impedances applied at both cable ends were chosen equal: $1 \mathrm{nF}$, $10 \mathrm{nF}$ (typical for load by transformer); $10 \Omega$ and $100 \Omega$ (typical for load by leaving cables). The bold horizontal line elements, representing the detection level of the transmitter/receiver unit available, should only be considered as indicative, since the performance depends on technical details of the equipment, which can be optimized further for the coupling and detection method used.

From the results it can be concluded that capacitive coupling clearly performs better in the sense of signal level. However, as argued in Section I, inductive coupling may still be preferred because it does not require galvanic contact with the phase conductors. Inductive coupling on both sides within the CENELEC $A$-band may be problematic, especially when only transformers terminate the cable. The ratio between efficiency of inductive and capacitive coupling for termination by transformers at both cable ends is given by

$$
\left|\frac{H_{i-i}}{H_{c-c}}\right|=\frac{M_{t}}{C_{t}} \cdot \frac{1}{\left|Z_{t} Z_{r}\right|} \sim \omega^{2}
$$




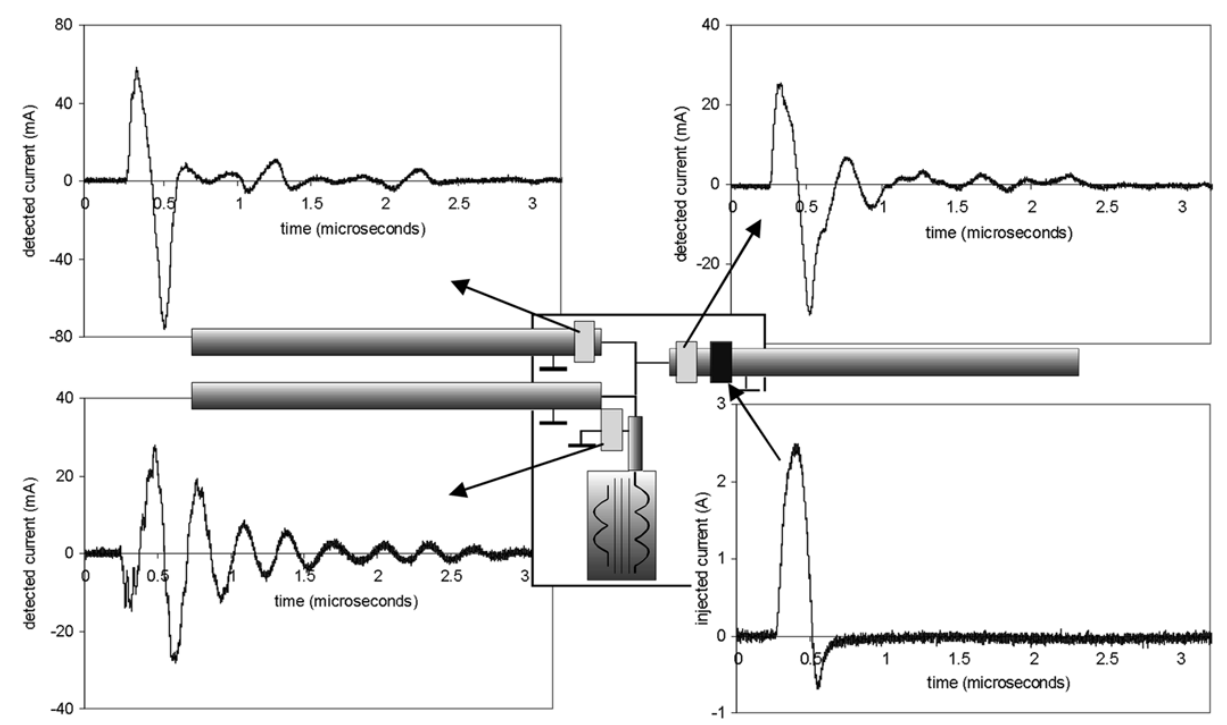

Fig. 11. Inductively injected pulse (right-bottom) over a PILC cable earth screen and measured waveforms at three positions, namely at the earth screen of the same incoming cable (right-top), a leaving cable (left-top) and the ground connection of the cable connection to the distribution transformer (left-bottom).

Inductive coupling therefore would profit strongly if higher frequency bands would be available for power line communication. Also, using coils with magnetic cores is an option to improve the coupling strength, but this will complicate installation and is relatively expensive (and should not saturate because of a $50-\mathrm{Hz}$ power current). It must be noted, that there will often be another cable as part of the termination impedance resulting in a more favorable injection and detection circuit.

\section{PULSE INJECTION}

Inductive pulse injection, required for time-base alignment in case of double-sided measurement (see Section I), can also be used for determining the relevant substation impedances. Since the injection coil and the substation circuit are weakly coupled, the corresponding circuits can be described independently: the primary injection current results in a known induced voltage over the impedances present in the secondary substation circuit. By measuring the secondary current these impedances can in principle be evaluated.

A well-defined pulse, with its energy mainly concentrated in the low $\mathrm{MHz}$ range, can be realized by discharging a capacitor over the induction of the injection coil. The resulting oscillation is blocked after a half cycle. Practical values are a $1 \mathrm{nF}$ capacitor charged to the order of $100 \mathrm{~V}$, and a $1 \mu \mathrm{H}$ inductance. The signal amplitude upon injection into the cable is kept below $1 \mathrm{~V}$. In Fig. 11 the results on a life substation are shown. For injection, a Rogowski coil with a mutual inductance of $M=42 \mathrm{nH}$ was mounted around the screen, beyond the last earth connection of an incoming cable. Detection was accomplished by means of commercially available current probes (1 V/A) at various positions:

- Sensor is positioned around the same cable as the injection coil. The measured signal is basically the time derivative of the injected current with the amplitude dependent on the substation impedance.
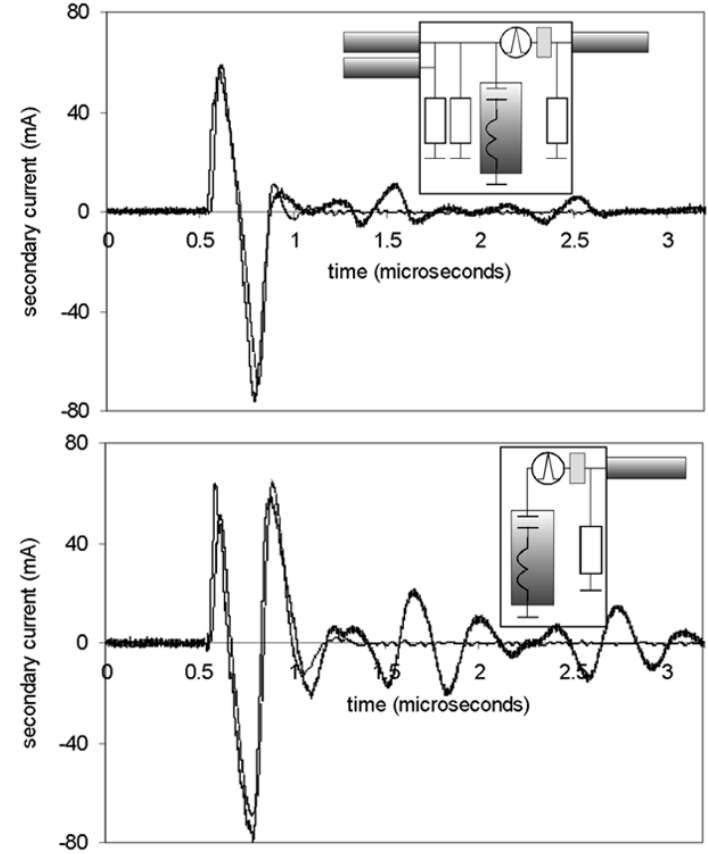

Fig. 12. Detected (bold line) and calculated (thin line) response upon the injected current for two situations: substation with a distribution transformer, one incoming and two leaving cables (top); substation with only a distribution transformer and an incoming cable (bottom). Cable characteristic impedances are indicated with resistor symbols, the transformer and its connection as a capacitor in series with a self-inductance.

- Sensor is positioned around a leaving cable. This signal resembles the former one, however the amplitude is reduced, since there are two similar leaving cables.

- Sensor is positioned around the ground connection of the cable to the distribution transformer. This path basically consists of a capacitance in series with the self-inductance of the connection.

From the measured secondary waveforms, and the known injection current $I_{\mathrm{inj}}$, the values of the relevant lumped impedances can be deduced. For two substations this is illustrated in 
Fig. 12. In both cases the secondary current is measured over the same cable screen as injection takes place. The secondary current $I_{\text {sec }}$ can be calculated from

$$
I_{\mathrm{sec}}=\frac{j \omega M}{Z_{\mathrm{inj}}+\left(\sum 1 / Z_{i}\right)^{-1}} I_{\mathrm{inj}}
$$

where $Z_{\text {inj }}$ represents the impedance of the cable containing the current probes; the summation in the denominator includes all impedances closing the current path. The substations shown in Fig. 12 differ in two additional leaving cables present in the top figure. The fits were obtained by using characteristic impedances of $12 \Omega$ (for the SP channel, consisting of three parallel phases with respect to ground) and using a total value of $3 \mathrm{nF}$ for the transformer $(1 \mathrm{nF})$ and its connecting cable $(2 \mathrm{nF})$. Further, the self-inductances of the connections were typically of the order of $0.5 \mu \mathrm{H}$ and an extra circuit resistance of about $4 \Omega$ was introduced to take into account the skin effect and radiation losses in order to get good agreement.

\section{DISCUSSION AND CONCLUSION}

Power line communication over the MV grid as channel for general purposes is unlikely to become widespread, as long as the maximum data transfer is limited by the current standards. Communication of data concerning the grid performance itself is more likely, since the required data rate is not high. As diagnostic tools are developed further and the condition of the grid and its components can be estimated more and more reliably, the availability of MV power line communication may become commercially interesting. For instance, in The Netherlands about $90000 \mathrm{~km}$ PILC cable is in use with a total value in the order of 5 billion Euros.

Any extension of the lifespan of some critical cable connections by proper monitoring, e.g., using two-sided PD detection with a communication channel in between, will save investment costs. The total cost of introducing these systems is clearly not determined solely by the cost of the equipment. Installation can be quite expensive if it would involve modifications to the substations or would require temporal disconnection of the cables. Commercial success will depend strongly on the simplicity of installation. Coupling schemes for signals to the MV cable should therefore be optimized not only for the technical (telecommunication) specifications, but should also include measures to allow easy, fast, and safe installation, preferably in life circuits. For the present purpose of PD diagnostics this implies that sensor tasks (PD-detection, pulse injection, and communication) should be integrated. The optimal bandwidths for detecting PDs and for pulse injection are basically determined by the propagation characteristics of the power cable, and are therefore similar. Communication is restricted by the standards. As discussed in Section VI, in a few situations inductive coupling efficiency may be insufficient for reliable data transfer. Alternative modulation schemes are under consideration, which still meet the standards. For instance, the pulse injection system can be applied to generate successive pulses (repetition rate within the CENELEC $A$-band) with modulated time differences or amplitudes. This option has the additional advantage that the communication device is directly integrated, and one does not have to rely on the presence of other communication channels.

\section{ACKNOWLEDGMENT}

The authors gratefully acknowledge A. van Staalduinen and T. Wilmes (TUE) for the Rogowski coils and assistance with the transformer measurements; $H$. van der Zanden and A. van Iersel (TUE) for the pulse injection system; C. Verhoeven and P. van Roij (ESSENT) for making a $4 \mathrm{~km}$ cable connection available; and M. van Riet (NUON) and M. Kruithof (REMU) for arranging the noise level measurements in substations.

\section{REFERENCES}

[1] P. A. A. F. Wouters, P. C. J. M. van der Wielen, and E. F. Steennis, "Challenges related to development of an on-line PD detection and localization system," in Proc. Nordic Insul. Symp., Tampere, Finland, Jun. 11-13, 2003, pp. 3-10.

[2] H. E. Orton, "Diagnostic testing of in-situ power cables-An overview," in Proc. 13th Int. Symp. High Voltage Eng., Delft, The Netherlands, Aug. 25-29, 2003.

[3] M. Michel, "Moving toward a complete on-line condition monitoring solution," in Proc. 17th CIRED, Barcelona, Spain, May 12-15, 2003.

[4] P. C. J. M. van der Wielen and P. A. A. F. Wouters, "Sensors for on-line PD detection in MV power cables and their locations in substations," in Proc. 7th Int. Conf. Properties Applicat. Dielect. Materials, Nagoya, Japan, Jun. 1-5, 2003, pp. 215-219.

[5] P. C. J. M. van der Wielen, E. F. Steennis, and P. A. A. F. Wouters, "Fundamental aspects of excitation and propagation of on-line partial discharge signals in three-phase medium voltage cable systems," IEEE Trans. Dielect. Elect. Insulation, vol. 10, no. 4, pp. 678-688, Aug. 2003.

[6] C. R. Paul, "Decoupling the multiconductor transmission line equations," IEEE Trans. Microw. Theory Tech., vol. 44, no. 8, pp. 1429-1440, Aug. 1996.

[7] A. R. Djordjević and T. K. Sarkar, "Analysis of time response of lossy multiconductor transmission line networks," IEEE Trans. Microw. Theory Tech., vol. MIT-35, no. 10, pp. 898-907, Oct. 1987.

[8] O. G. Hooijen, "A channel model for the residential power circuit used as a digital communications medium," IEEE Trans. Electromagn. Compat., vol. 40, no. 4, pp. 331-336, Nov. 1998.

[9] P. T. M. Vaessen and E. Hanique, "A new frequency response analysis method for power transformers," IEEE Trans. Power Del., vol. 7, no. 1, pp. 384-391, Jan. 1992.

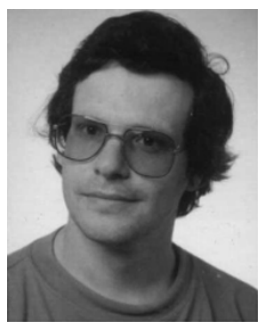

Peter A. A. F. Wouters was born in Eindhoven, The Netherlands, on June 9, 1957. He studied physics at the Utrecht University (UU), Utrecht, The Netherlands, until 1984, from which he received the Ph.D. degree for a study on elementary electronic transitions between metal surfaces and low energetic (multiple) charged ions in 1989.

In 1990, he joined the Electrical Power Systems (EPS) group at the Technical University of Eindhoven, Eindhoven, The Netherlands, as Research Associate. His research interests include partial discharge techniques, vacuum insulation, and LF electromagnetic field screening. 


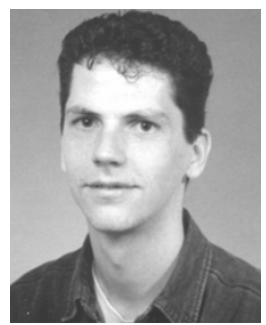

Peter C. J. M. van der Wielen (S'93) was born in Hulst, The Netherlands, on March 6, 1973. He studied electrical engineering at the Eindhoven University of Technology (EUT), Eindhoven, The Netherlands, where he was member of the board and several committees of the IEEE Student Branch Eindhoven. He received the M.Sc. degree in 2000. Since then, he has been pursuing the Ph.D. degree, doing a study on power cable diagnostics at both the Electrical Power Systems group at EUT and KEMA, Arnhem, The Netherlands, Transmission and Distribution Testing Services. His study primarily deals with different aspects related to online partial discharge (PD) monitoring of medium-voltage power cable systems, including cable and substation modeling, sensors, PD localization, measurement setup synchronization, and communication.

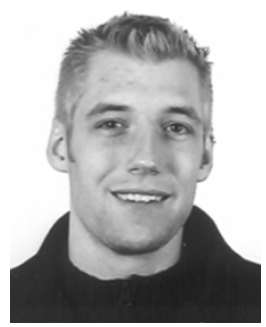

Jeroen Veen ( $\left.\mathrm{S}^{\prime} 00\right)$ was born in Dordrecht, The Netherlands, on September 20, 1977. He received the B.Eng. in telematics from the University of Professional Education, Utrecht, The Netherlands, in 1998 and the M.Sc. (Honors) in electrical engineering from the Eindhoven University of Technology, Eindhoven, The Netherlands, in 2001. Currently he is pursuing the Ph.D. degree from the Eindhoven University of Technology, Signal Processing Systems (SPS) group, and KEMA, Arnhem, The Netherlands, Transmission and Distribution Testing Services. His main research activities lie in signal processing techniques applied to online partial discharge monitoring of medium-voltage power cable systems.

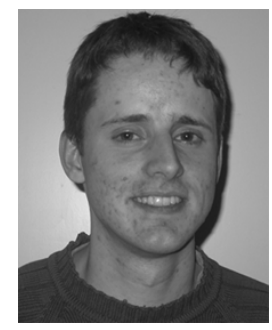

Paul Wagenaars was born in Schaijk, The Netherlands, on February 1, 1981. He has been studying electrical engineering since 1999 at the Eindhoven University of Technology, Eindhoven, The Netherlands, where he is pursuing the M.Sc. degree at the Electrical Power Systems (EPS) group. His M.Sc. project is deals with sensors for online partial discharge monitoring of medium-voltage power cable systems.

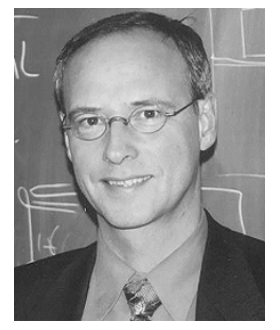

E. Fred Steennis joined KEMA, Arnhem, The Netherlands, in 1982, after his education at the Technical University in Eindhoven, Eindhoven, The Netherlands, where he studied degradation mechanisms in energy cables for the Dutch utilities. It was on this subject that he received the doctors thesis from the Technical University in Delft, Delft, The Netherlands, in 1989.

In and outside the Netherlands, he is a consultant on energy cables and is the author and a teacher of the KEMA course on Power Cables. At KEMA, he is the Technical Manager of the Department of High-Voltage Testing and Diagnostics. Since 1999, he has also been a part-time Professor at the Technical University in Eindhoven, where he teaches and studies diagnostics for power cables.

Dr. Steenis received the Hidde Nijland award for his contributions in 1991 After that, his experience on degradation mechanisms and related test methods both in the field and the laboratory was further enhanced. Based on this expertise, he became the Dutch representative with the Cigre Study Committee on High-Voltage Cables. He is also a member of various international Working Groups. 